\title{
The influence of advanced outlines on the free recall of prose
}

\author{
ANDREW D. COHEN and ARTHUR C. GRAESSER \\ California State University, Fullerton, California 92634
}

\begin{abstract}
This study examined how the free recall of prose is affected by (1) outlines as advanced organizers, (2) preexperimental familiarity with the material, and (3) text genre, that is, narrative vs. expository prose. College students studied a two-level outline, a one-level outline, or no outline before listening to 400 - to 500 -word passages that varied in familiarity and narrativity. Subjects later recalled the passages and then generated their own subjective outline of each passage. Overall, the outline manipulation did not significantly influence recall. However, significant negative correlations were found between the amount recalled and the degree of subjective-experimental outline correspondence. These outcomes are discussed in the context of interference theory, a depth-of-processing framework, and an automatic comprehension hypothesis. Whereas familiarity had no significant effect on recall, text genre had a robust effect. Less information was recalled from expository passages than from narrative passages.
\end{abstract}

Numerous studies have explored how advanced organizers influence memory, but there have been mixed outcomes. Advanced organizers are outliners, diagrams, or other forms of information that are presented before the material to be learned. Ausubel's early research supported the notion that advanced organizers facilitate retention. Ausubel $(1960,1962)$ proposed that a cognitive structure is produced by the advanced organizer and that the structure guides the organization of the material to be learned. However, since Ausubel's early research and theory, studies conducted in this paradigm have failed to support the intuitively compelling prediction. In a review of 32 of these studies (Barnes \& Clawson, 1975), only 12 (38\%) were found to support Ausubel's theory.

Available theories in cognitive psychology, information processing, and verbal learning would not necessarily predict that advanced organizers would facilitate acquisition and retention. For example, it is possible that prose comprehension is guided by highly organized processes that are overlearned after years of practice. It is predicted that the effects of advance organizers would be overridden by this automatized processing mechanism. An automatic comprehension hypothesis proposes that advanced organizers would not lead to a significant improvement on learning and comprehension of prose when the prose is easy to comprehend.

Other theories that would also explain the fact that advanced organizers are ineffective are the the depth-ofprocessing framework (Craik \& Lockhart, 1972) and traditional interference theory (Postman \& Underwood, 1973). According to the interference theory, the content of the advance organizer may interfere with the content of the acquisition material. A plausible prediction of interference theory is that interference should increase as the content of the advanced organizer deviates more and more from the content of the passage. A depth-ofprocessing framework might make the opposite pre- diction; when there is a substantial correspondence between the outline and the passage, the comprehender should process the material less deeply, which should lead to poorer acquisition and retention of the acquisition material.

In a study by Graesser, Hauft-Smith, Cohen, and Pyles (in press), outlines were sometimes presented to subjects prior to their listening to passages. In other conditions, no outline was given. The advanced outlines were not found to facilitate retention of the passages. This outcome would seem to support the automatic comprehension hypothesis. However, in that study, there was no assessment of the degree to which the advanced outline overlapped with the subjective organization of the passages constructed by the subjects. In order to determine whether traditional interference theory or the depth-of-processing framework is correct, it is important to assess this degree of correspondence. This correspondence was directly measured in the present study.

In the Graesser, Hauft-Smith, Cohen, and Pyles (in press) study, acquisition and retention of material was assessed by either a cued recall test or a three-alternative forced-choice (3AFC) test. Given that memory performance is highly sensitive to the type of testing procedures (Bransford, 1979; Gagne, 1978), the present study collected free recall protocols for assessing the subject's memory for the passages. There are reasons to believe that an advanced outline may have a greater effect on a free recall task because the subjects are required to organize their retrieval strategies. In contrast, cued recall and $3 \mathrm{AFC}$ tests do not require these organizational abilities to the same extent.

\section{METHOD}

\author{
Subjects \\ Twelve students at California State University, Fullerton, \\ received $\$ 10$ for participating in this experiment.
}




\section{Materials}

The 12 passages used in the Graesser et al. (in press) study were recorded on a tape recorder at a medium rate of 150 words $/ \mathrm{min}$. The passages were approximately $400-500$ words long. The 12 passages were exemplars of four different passage categories: familiar narrative, unfamiliar narrative, familiar expository, and unfamiliar expository. Each category contained three passages. The titles for familiar narrative passages were Noah's Ark, Snow White, and The Story of Jonah; for unfamiliar narrative passages they were Serpent Story, Story of Bodisat, and The Princess and the Pea; for familiar expository passages, they were Earthquakes, Emotions, and The Earth's Orbit; and for unfamiliar expository passages, they were Coal Energy, Harvester Ants, and Armadillos.

The 12 passages were rated on familiarity by subjects in the Graesser, Hauft-Smith, Cohen, and Pyles (in press) study, using a 7-point scale where $1=$ "not at all familiar with the information in the passage" and 7= "very familiar with the information in the passage." There also was a narrativity rating for each passage, which was based on a 7-point scale ranging from $1=$ "passage conveys static information" to $7=$ "passage conveys active information with events unfolding in time." The mean familiarity ratings for familiar narrative, unfamiliar narrative, familiar expository, and unfamiliar expository passages were $4.21,3.00$, 4.54 , and 2.54 , respectively. The mean narrativity ratings for familiar narrative, unfamiliar narrative, familiar expository, and unfamiliar expository passages were $6.08,6.38,2.25$, and 2.26 , respectively.

The four passage categories were presented in blocks, with three passages per block. The presentation order of passage categories was counterbalanced by a 4 by 4 Latin square, with three subjects per ordering.

For each passage, outlines were constructed with two levels of information: a main level and a secondary level. The main level included two to seven statements that were not explicitly stated in the passage. For each main level statement, there were two to four secondary level statements. A one-level outline contained only the main statements, whereas a two-level outline contained both main and secondary statements. For any given subject, one of the three passages within a passage category had no outline, another had a one-level outline, and the other had a two-level outline. Each subject received one of three outline presentation orderings $(012,120$, or 201$)$, which were assigned to the three passages within each of the four passage categories. Thus, subjects received each outline condition within each passage category.

Booklets were prepared in such a way that subjects received a number of pages for each passage: (1) a one-, two- or zerolevel outline on the first page, (2) a blank page for recall of the presented outline, (3) two blank pages for passage recall, and (4) one blank page for generation of a subjective outline.

\section{Procedure}

Subjects were told that they would be studying passages and then recalling them. They were told that sometimes they would receive an outline before the passage. Subjects studied the outline in their booklet for $1.5 \mathrm{~min}$. The subjects then turned to the next page, which was blank, and wrote down as much of the outline as they could remember. The outline recall was completed to insure that the subjects acquired the outline. The subjects were then instructed to go back to the outline and refer to it while listening to the passage. After the passage presentation, the subjects turned to the third page of the booklet, which was blank, and wrote down as much of the passage as they could recall. After $15 \mathrm{~min}$, the subjects turned to the next page and wrote an outline of the passage they had just recalled, using their own idea of what concepts should be part of the passage outline. The subjects were told that it was possible that their own outline would be similar to the presented outline. It should be noted that no outline had been presented in the zero-level outline condition. When the subjects completed writing their outline, they continued to the next passage.

\section{RESULTS}

In order to analyze the amount of information recalled, each story was divided into propositions. Among the 12 passages, the mean number of propositions was 38 , ranging from 29 to 53 . A sentence was scored as recalled if the gist or meaning of the sentence was captured. Recall proportions were computed by dividing the number of propositions recalled by the number of propositions in a passage.

An analysis of variance was performed on recall proportions as a function of narrativity, familiarity, and outline level. The means of this analysis are shown in Table 1 . In order to test whether effects were statistically significant, we used Clark's (1973) $\min \mathrm{F}^{\prime}$ test. This is a conservative test, with an error term that is based on both subject variance and item (passage) variance.

Recall was shown to be significantly higher for narrative passages than expository passages $\left[\min \mathrm{F}^{\prime}(1,16)=\right.$ $60.80, \mathrm{p}<.01]$. The mean recall proportion was .62 for narrative passages and .35 for expository passages. There were no significant main effects for familiarity and outline level. The mean recall proportion for familiar passages was .46 and .52 for unfamiliar passages. The mean recall proportions in the zero-level, one-level, and two-level outline conditions were $.47, .49$, and .51 , respectively. There were no significant interactions.

In order to obtain a measurement of the degree to which the subject's natural subjective outline corresponded to the experimental outline, a subjectiveexperimental outline correspondence score (SEC) was computed. The subjective outlines generated from the subjects presented with no outline were used to compute SEC scores. SEC scores were calculated by dividing the number of subjective outline statements, which were also experimental outline statements, by the number of statements in the experimental outline. Separate SEC scores were computed for one- and two-level outlines.

A measurement of outline facilitation was computed by dividing the recall proportions of those subjects who received the experimental outline by the recall proportions of those subjects who received no outline. Outline facilitation scores were computed for one- and two-level outlines, separately.

Correlations between SEC scores and outline facilitation scores were obtained for both one- and two-level outlines. For one-level outlines, the correlation was significantly negative $(r=-.55, p<.05)$. There was

Table 1

Mean Proportion of Passage Propositions Recalled

\begin{tabular}{lccc} 
& \multicolumn{3}{c}{ Outline Level } \\
\cline { 2 - 4 } Passage Category & No Outline & One & Two \\
\hline Narrative Familiar & .56 & .54 & .59 \\
Narrative Unfamiliar & .68 & .68 & .67 \\
Expository Familiar & .31 & .35 & .40 \\
Expository Unfamiliar & .31 & .38 & .37 \\
\hline
\end{tabular}


also a significant negative correlation between outline facilitation and SEC scores for two-level outlines $(r=$ $-.67, p<.05$. Thus, for both levels of outline, there were significantly negative correlations between SEC and outline facilitation. The higher the correspondence between subjective and experimental outlines, the less the outline facilitated recall. A median split of SEC scores showed $67 \%$ of the low-SEC scores had positive outline facilitation, whereas $63 \%$ of the high-SEC scores had negative outline facilitation.

\section{DISCUSSION}

The procedures and analyses in this study revealed more about the effects of advanced outlines on retention of prose. There were no overall effects of outlines on recall of the passages. This finding agrees with other studies and reviews (Barnes \& Clawson, 1975; Graesser, Hauft-Smith, Cohen, \& Pyles, in press), and it seems to be problematic to Ausubel's (1960) proposal. However, the fact that no overall effects were found does not imply that outlines are completely ineffective. The results of the present study show an interesting, but more complex relationship. Some outlines facilitated recall of prose, and others interfered. The net effect was no effect. If there is a large degree of overlap between the experimental outline and the subjective organization of the passages that subjects normally construct, then the outline interferes with acquisition of the passages. If, however, there is some deviation between the experimental outline and the subjective organization of the passage, then acquisition of the passage is facilitated. The data would seem to support the depth-of-processing framework. The subjects processed the passages at greater depths to the extent that there was a discrepancy between the outline and the passage. The above trends suggest a need to understand the interactions between the outline representation and the passage representations in order to predict how an outline will affect retention of the material to be learned.

Recall was not higher for passages on familiar topics than unfamiliar topics. In fact, the trend was just the opposite, although not significant. This finding replicated the data in the Graesser, Hauft-Smith, Cohen, and Pyles (in press) study and other studies (Gagne, 1978; Kalbaugh \& Walls, 1973). However, this trend may not hold over longer intervals. Whereas unfamiliar passages are recalled better than familiar passages after short retention intervals, the opposite trend emerges after long retention intervals (Gagne, 1978).

As in the Graesser, Hauft-Smith, Cohen, and Pyles (in press) study, it was found that prose genre robustly predicted the amount of material recalled. Recall for narrative prose was roughly twice as good as for expository prose. This finding emerges in a variety of testing formats, including free recall, prompted recall, and comprehension tests. The fact that narrative prose is remembered better than expository prose cannot be attributed to the amount of time someone studies the material. A reading time study by Graesser, Hoffman, and Clark (1980) showed that subjects spend more time reading expository than reading narrative prose. Therefore, less time is needed to comprehend narrative prose, and yet narrative is remembered better.

The data in this study showed how important it is to study how material to be learned is represented and organized. The same holds for devices that are imposed to facilitate acquisition and retention. Without such an understanding, one cannot predict when learning devices will facilitate acquisition and what type of material is easy to acquire.

\section{REFERENCES}

Ausubel, D. P. The use of advanced organizers in learning and retention of meaningful verbal material. Journal of Educational Psychology, 1960, 51, 267-272.

Ausubel, D. P., \& Fitzgerald, D. Organizer, general background, and antecedent learning variables in sequential verbal learning. Journal of Educational Psychology, 1962, 53, 243-249.

Barnes, B. R., \& Clawson, E. V. Do advanced organizers facilitate learning? Recommendations for further research based on an analysis of 32 studies. Review of Educational Research, 1975, 45, 637-659.

Bransford, J. D. Human cognition: Learning, understanding and remembering. Belmont, Calif: Wadsworth, 1979.

Clark, H. H. The language-as-fixed-effect fallacy: A critique of language statistics in psychological Research. Journal of Verbal Learning and Verbal Behavior, 1973, 12, 335-359.

Craik, F. I. M., \& LockharT, R. S. Levels of processing: A framework for memory research. Journal of Verbal Learning and Verbal Behavior, 1972, 11, 671-684.

GAGNE, E. D. Long-term retention of information following learning from prose. Review of Educational Research, 1978, 48, 629-665.

Graesser, A. C., Hauft-Smith, K., Cohen, A. D., \& Pyles, L. D. Advanced outlines, familiarity, text genre and retention of prose. Journal of Experimental Education, in press.

Graesser, A. C., Hoffman, N. L., \& Clark, L. F. Structural components of reading time. Journal of Verbal Learning and Verbal Behavior, 1980, 19, 135-151.

Kalbaugh, G. L., \& Walls, R. T. Retroactive and proactive interference in prose learning of biographical and science material. Journal of Educational Psychology, 1973, 65, 244-251.

Postman, L., \& Unde Rwood, B. J. Critical issues in interference theory. Memory \& Cognition, 1973, 1, 19-40.

(Received for publication March 27, 1980.) 